\title{
Smarandache curves of Anti-Salkowski curve according to the spherical indicatrix curve of the unit darboux vector
}

Anti-Salkowski ĕgrisinin birim darboux vektöründen elde edilen smarandache ĕgrileri

\author{
Süleyman ŞENYURT ${ }^{* 1, a}$, Melek UZUN ${ }^{1, b}$ \\ ${ }^{I}$ Ordu University, Faculty of Arts and Sciences, Department of Mathematics, 52200, Ordu-Turkey
}

\begin{abstract}
In this paper, we have defined special Smarandache curves according to Sabban frame formed by the unit Darboux vector of Anti-Salkowski curve. Next, the Sabban frame belonging to these curves have been constituted. Last, the geodesic curvatures of these Smarandache curves have been calculated and an example for each curve has been illustrated.
\end{abstract}

Keywords: Anti-Salkowski curve, Sabban frame, Smarandache curves, Unit darboux vector

$\ddot{\partial} z$

Bu çalışmada, Sabban çatısına göre anti-Salkowski eğrisinin birim Darboux vektörlerinden elde edilen özel Smarandache ĕgrileri tanımlandı. Daha sonra her bir Smarandache ĕgrisinin Sabban çatısı oluşturuldu. Son olarak bu Smarandache ĕgrilerinin geodezik ĕgrilikleri hesaplandı ve her bir ĕgriye ait grafikler çizildi.

Anahtar kelimeler: Anti-Salkowski ĕgrisi, Saban çatısl, Smarandache eğrisi, Birim darboux vektörü

\footnotetext{
*a Süleyman ŞENYURT; senyurtsuleyman52@gmail.com; Tel: (0452) 2265200; orcid.org/0000-0003-1097-5541

${ }^{\mathrm{b}}$ orcid.org/0000-0002-1598-1345
} 


\section{Introduction}

In 1909, Erich Salkowski defined curve families with non-constant $\tau$ and constant curvature $\kappa$ in (Salkowski, 1909). Later J. Monterde constructed a method for closed curves and the properties of Salkowski and anti-Salkowski curve used in (Monterde, 2008). In 1990, the geodesic curve of a spherical curve is calculated by J. Koenderink with the Sabban frame of the spherical indicatrix curves in (Koenderink, 1990).

Then the Smarandache curves obtained from Sabban frame are defined and geodesic curvatures of these curves are given in (Gür and Şenyurt,2010; Şenyurt and Öztürk, 2018a,b; Uzun and Şenyurt, 2020).

In this study, Smarandache curves are defined according to the Sabban frames belonging to the spherical indicatrix curves of each of the $\{T, N, B\}$, Frenet vectors of the anti-Salkowski curve. The geodesic curvatures of these curves are then calculated.

\section{Materials and methods}

Let $\alpha: I \rightarrow E^{3}$ be a unit speed curve, we defined the quantities of the Frenet apparatus and Frenet formulae, respectively (Sabuncuoğlu, 2006),

$$
\begin{aligned}
& T_{\alpha}=\alpha^{\prime}, N_{\alpha}=\frac{\alpha^{\prime \prime}}{\left\|\alpha^{\prime \prime}\right\|}, B_{\alpha}=T_{\alpha} \wedge N_{\alpha} \\
& T_{\alpha}^{\prime}=\kappa_{\alpha} N_{\alpha}, N_{\alpha}^{\prime}=-\kappa_{\alpha} T_{\alpha}+\tau_{\alpha} B_{\alpha}, B_{\alpha}^{\prime}=-\tau_{\alpha} N_{\alpha} .
\end{aligned}
$$

If the Frenet vectors are computed as arbitrary parameter, we can write

$$
\begin{aligned}
& T_{\alpha}=\frac{\alpha^{\prime}}{\left\|\alpha^{\prime}\right\|}, N_{\alpha}=T_{\alpha} \wedge B_{\alpha}, B_{\alpha}=\frac{\alpha^{\prime} \wedge \alpha^{\prime \prime}}{\left\|\alpha^{\prime} \wedge \alpha^{\prime \prime}\right\|}, \\
& \kappa_{\alpha}=\frac{\left\|\alpha^{\prime} \wedge \alpha^{\prime \prime}\right\|}{\left\|\alpha^{\prime}\right\|^{3}}, \tau_{\alpha}=\frac{\operatorname{det}\left(\alpha^{\prime}, \alpha^{\prime \prime}, \alpha^{\prime \prime \prime}\right)}{\left\|\alpha^{\prime} \wedge \alpha^{\prime \prime}\right\|^{2}}
\end{aligned}
$$

(Sabuncuoğlu, 2006). The Frenet-Serret axis system, moving with the point, has an angular velocity. Dividing this by the (signed) point speed, that is, taking the derivative of the angular position of the axis system with respect to the path position, gives the Darboux vector, $W_{\alpha}$ which is given in value by

$$
W_{\alpha}=\tau_{\alpha} T_{\alpha}+\kappa_{\alpha} B_{\alpha}
$$

The unit Darboux vector is

$$
C_{\alpha}=\sin (\theta) T_{\alpha}+\cos (\theta) B_{\alpha}, \quad \cos (\theta)=\frac{\kappa_{\alpha}}{\sqrt{\kappa_{\alpha}{ }^{2}+\tau_{\alpha}^{2}}}, \quad \sin (\theta)=\frac{\tau_{\alpha}}{\sqrt{\kappa_{\alpha}{ }^{2}+\tau_{\alpha}^{2}}}
$$

where $\measuredangle(B, W)=\boldsymbol{\theta}$ (Fenchel, 1951).

Let $\gamma: I \rightarrow S^{2}$ be a unit speed spherical curve. We denote $S$ as the arc-length parameter of $\gamma$. Let us denote by

$\gamma(s)=\gamma(s), t(s)=\gamma^{\prime}(s), d(s)=\gamma(s) \wedge t(s)$

$\{\gamma(s), t(s), d(s)\}$ frame is called the Sabban frame of $\gamma$ on $S^{2}$. Then we have the following spherical Frenet formulae of $\gamma$

$\gamma^{\prime}(s)=t(s), \quad t^{\prime}(s)=-\gamma(s)+\kappa_{g}^{\gamma}(s) d(s), \quad d^{\prime}(s)=-\kappa_{g}^{\gamma}(s) t(s)$

where $\kappa_{g}$ is called the geodesic curvature of the curve $\gamma$ on $S^{2}$ which is

$\boldsymbol{\kappa}_{g}^{\gamma}(s)=\left\langle t^{\prime}(s), d(s)\right\rangle($ Koenderink,1990). 
Definition 2.1 (Anti-Salkowski curve) For any $m \neq \pm \frac{1}{\sqrt{3}}, 0$ with $\mathrm{n}=\frac{m}{\sqrt{1+m^{2}}}, m \in \mathbb{R}$, let us define the space curve

$$
\beta_{m}(t)=\left(\begin{array}{l}
\frac{n}{2\left(4 n^{2}-1\right) m}\left(n\left(1-4 n^{2}+3 \cos (2 n t)\right) \cos (t)+\left(2 n^{2}+1\right) \sin (t) \sin (2 n t)\right) \\
\frac{n}{2\left(4 n^{2}-1\right) m}\left(n\left(1-4 n^{2}+3 \cos (2 n t)\right) \sin (t)-\left(2 n^{2}+1\right) \cos (t) \sin (2 n t)\right), \\
\frac{n^{2}-1}{4 n}(2 n t+\sin (2 n t))
\end{array}\right)
$$

(Salkowski,1909). Frenet apparatus are

$$
\left\{\begin{array}{l}
T=-\left(\cos (t) \sin (n t)-n \sin (t) \cos (n t), \sin (t) \sin (n t)+n \cos (t) \cos (n t), \frac{n}{m} \cos (n t)\right) \\
N=n\left(\frac{\sin (t)}{m},-\frac{\cos (t)}{m}, 1\right) \\
B=\left(-\cos (t) \cos (n t)-n \sin (t) \sin (n t),-\sin (t) \cos (n t)+n \cos (t) \sin (n t), \frac{n}{m} \sin (n t)\right) \\
\kappa=\tan (n t), \tau=1,\left\|\beta_{m}^{\prime}(t)\right\|=\frac{\cos (n t)}{\sqrt{1+m^{2}}}
\end{array}\right.
$$

(Monterde, 2008).

\section{Results and discussion}

If we take $\kappa=\tan (n t)$ and $\tau=1$, from the equation (1) we can write

$$
\begin{aligned}
C(t) & =\frac{\tau}{\sqrt{\kappa^{2}+\tau^{2}}} T+\frac{\kappa}{\sqrt{\kappa^{2}+\tau^{2}}} B \\
& =\frac{1}{\sqrt{\tan ^{2}(n t)+1}} T+\frac{\tan (n t)}{\sqrt{\tan ^{2}(n t)+1}} B \\
C(t) & =\cos (n t) T+\sin (n t) B .
\end{aligned}
$$

(6) using the equation (5), we get

$$
C(t)=\left(\begin{array}{l}
-2 \sin (n t) \cos (n t) \cos (t)+n \sin (t) \cos (2 n t), \\
-2 \sin (n t) \cos (n t) \sin (t)-n \cos (t) \cos (2 n t),-\frac{n}{m} \cos (2 n t)
\end{array}\right) .
$$

Let $S^{2}$ be a unit sphere in Euclidean 3-space and suppose that the unit speed regular curve $\alpha_{C}(t)=C(t)$ lying fully on $S^{2}$. Differentiating the equation (6), with respect to $S$, we have

$\frac{d \alpha_{C}(s)}{d s} \frac{d s}{d t}=-n \sin (n t) T+\cos (n t) T^{\prime}+n \cos (n t) B+\sin (n t) B^{\prime}$ 
$T_{C} \frac{d s}{d t}=n(-\sin (n t) T+\cos (n t) B)$

where $\frac{d s}{d t}=n$. Thus, the tangent vector of curve $\alpha_{C}$ is to be

$T_{C}=-\sin (n t) T+\cos (n t) B$.

From the equation (5), we can write

$$
T_{C}=\left(\begin{array}{l}
-\cos (t) \cos (2 n t)-2 n \sin (n t) \sin (t) \cos (n t), \\
-\sin (t) \cos (2 n t)+2 n \cos (t) \cos (n t) \sin (n t), \frac{2 n}{m} \cos (n t) \sin (n t)
\end{array}\right) .
$$

Differentiating the equation (8) with respect to $S$, we get

$$
T_{C}^{\prime}=-\cos (n t) T-\frac{\sec (n t)}{n} N-\sin (n t) B .
$$

Considering the equations (6) and (8), it easily seen that

$$
C \wedge T_{C}=-N=-n\left(\frac{\sin (t)}{m},-\frac{\cos (t)}{m}, 1\right)
$$

From the equation (10) and (11), the geodesic curvature of $\alpha_{C}$ is

$$
\begin{aligned}
K_{g} & =\left\langle T_{C}{ }^{\prime}, C \wedge T_{C}\right\rangle \\
K_{g} & =\left\langle-\cos (n t) T-\frac{\sec (n t)}{n} N-\sin (n t) B,-N\right\rangle \\
K_{g} & =\frac{\sec (n t)}{n} .
\end{aligned}
$$

Let $\alpha_{C}(t)=C(t)$ be a unit speed spherical curve. We denote $s$ as the arc-length parameter of $\alpha_{C}$. Let us denote by $C=C(t), T_{C}=C^{\prime}(t), C \wedge T_{C}=C(t) \wedge T_{C}(t) .\left\{C, T_{C}, C \wedge T_{C}\right\}$ from is called Sabban frame of $\alpha_{C}$ on unit sphere. Then equation (3) we have following spherical Sabban formulae of $\alpha_{C}$ curve

$$
\begin{aligned}
& C^{\prime}=T_{C}, \quad T_{C}^{\prime}=-C+\kappa_{g}\left(C \wedge T_{C}\right), \quad\left(C \wedge T_{C}\right)^{\prime}=-\kappa_{g} T_{C}, \\
& C^{\prime}=T_{C}, \quad T_{C}^{\prime}=-C+\frac{\sec (n t)}{n}\left(C \wedge T_{C}\right), \quad\left(C \wedge T_{C}\right)^{\prime}=-\frac{\sec (n t)}{n} T_{C},
\end{aligned}
$$




$$
\left\{\begin{array}{l}
C^{\prime}=-\sin (n t) T+\cos (n t) B \\
T_{C}{ }^{\prime}=-\cos (n t) T-\frac{\sec (n t)}{n} N-\sin (n t) B \\
\left(C \wedge T_{C}\right)^{\prime}=\frac{1}{n}(\tan (n t) T-B)
\end{array}\right.
$$

Definition 3.1. Let $\alpha_{C}(t)=C(t)$ be an anti-Salkowski indicatrix curve and $\left\{C, T_{C}, C \wedge T_{C}\right\}$ be the Sabban frame of this curve. Then $\beta_{1}(t)$-Smarandache curve is given by

$$
\beta_{1}(t)=\frac{1}{\sqrt{2}}\left(C+T_{C}\right)
$$

Substituting $C$ and $T_{C}$ vectors into the equations (7) and (9), we get the curve $\beta_{1}(t)$ as follow (Figure 1.):

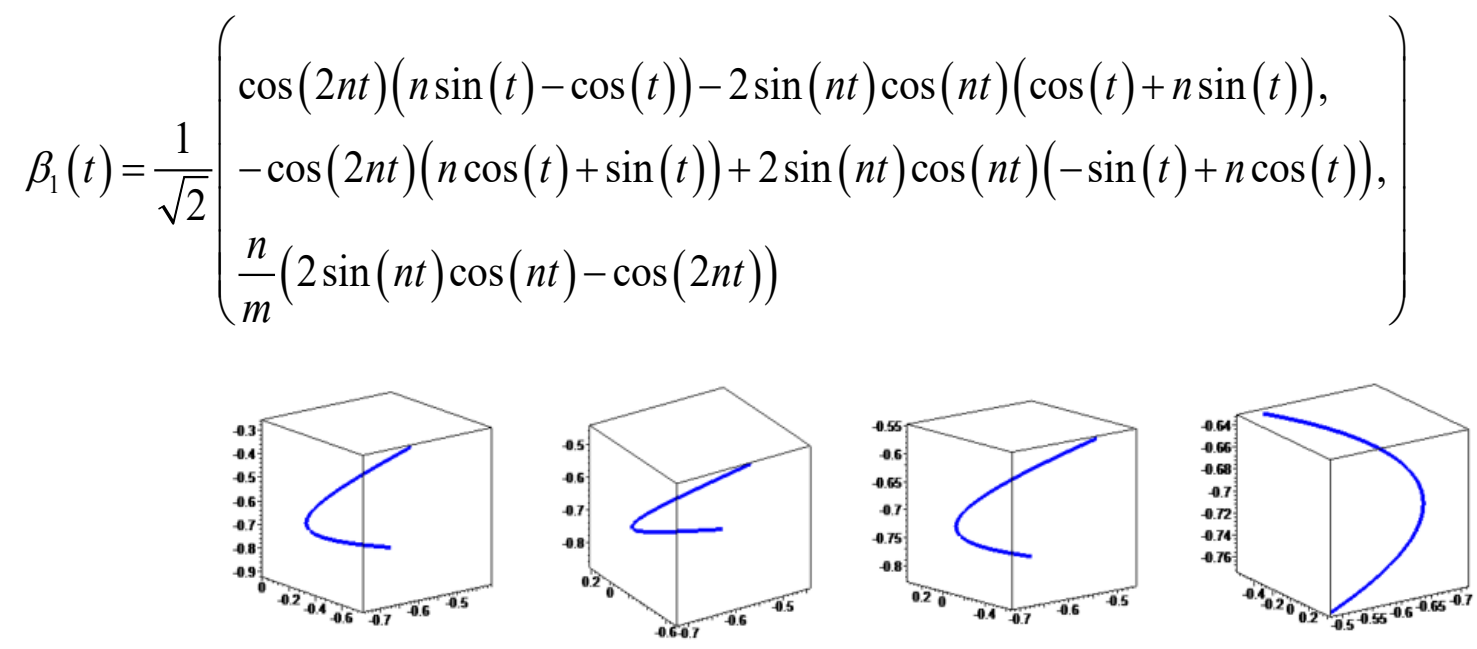
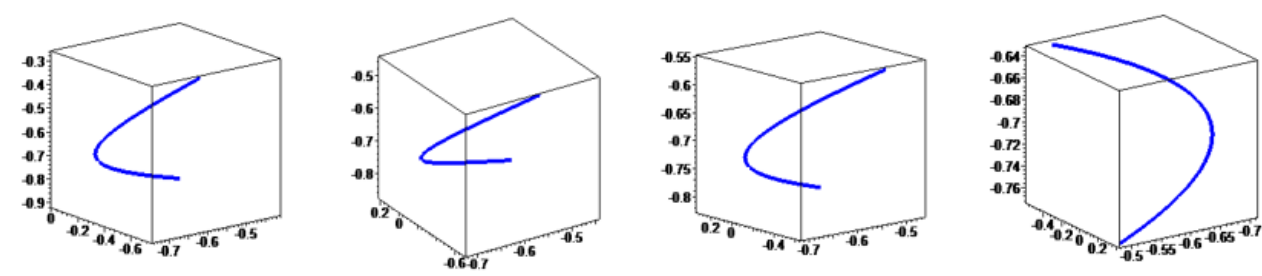

Figure 1. $\beta_{1}(t)$-Smarandache curves for $m=\frac{1}{3}, \frac{1}{5}, \frac{1}{8}, \frac{1}{16}$, respectively.

Theorem 3.1. The geodesic curvature $K_{g}{ }^{\beta_{1}}$ according to $\beta_{1}(t)$ - Smarandache curve is

$$
K_{g}^{\beta_{1}}=\frac{\sqrt{2}\left(\sec (n t)+4 n^{2} \cos (n t)+4 n^{4} \cos ^{3}(n t)+2 n^{5} \cos ^{2}(n t) \sin (n t)+n^{3} \sin (n t)\right)}{\cos ^{2}(n t)\left(2 n^{2} \cos ^{2}(n t)+1\right) \sqrt{\left(2 n^{2}+\sec ^{2}(n t)\right)}}
$$

Proof: Differentiating the equation (16), with respect to $S$, we have

$$
\begin{aligned}
& \frac{d \beta_{1}}{d s} \frac{d s}{d t}=\frac{1}{\sqrt{2}}\left(C^{\prime}+T_{C}^{\prime}\right), \\
& T_{\beta_{1}} \frac{d s}{d t}=\frac{-C+T_{C}+K_{g}\left(C \wedge T_{C}\right)}{\sqrt{2}},
\end{aligned}
$$


$T_{\beta_{1}}=\frac{-C+T_{C}+K_{g}\left(C \wedge T_{C}\right)}{\sqrt{2+K_{g}^{2}}}$

where $\frac{d s}{d t}=\frac{\sqrt{2+K_{g}^{2}}}{\sqrt{2}}$. If again derivative is taken, we get

$T_{\beta_{1}}^{\prime} \frac{d s}{d t}=\frac{\left(-C+T_{C}+K_{g}\left(C \wedge T_{C}\right)\right)^{\prime} \sqrt{2+K_{g}^{2}}-\left(\sqrt{2+K_{g}^{2}}\right)^{\prime}\left(-C+T_{C}+K_{g}\left(C \wedge T_{C}\right)\right)}{2+K_{g}^{2}}$,

$T_{\beta_{1}}^{\prime}=\frac{\sqrt{2}\left(\left(-2-K_{g}{ }^{2}+K_{g} K_{g}^{\prime}\right) C-\left(2+K_{g}{ }^{4}+3 K_{g}{ }^{2}+K_{g} K_{g}^{\prime}\right) T_{C}+\left(2 K_{g}+K_{g}{ }^{3}+2 K_{g}^{\prime}\right)\left(C \wedge T_{C}\right)\right)}{\left(2+K_{g}{ }^{2}\right)^{2}}$

Using the equations (6) and (19),we easily find

$C \wedge T_{\beta_{1}}=\frac{-K_{g} T_{C}+C \wedge T_{C}}{\sqrt{2+K_{g}^{2}}}$

From the equation (12), (20) and (21), the geodesic curvature of $\beta_{1}(t)$ is completed.

Definition 3.2. Let $\alpha_{C}(t)=C(t)$ be an anti-Salkowski indicatrix curve and $\left\{C, T_{C}, C \wedge T_{C}\right\}$ be the Sabban frame of this curve. Then Smarandache curve is given by

$\beta_{2}(t)=\frac{1}{\sqrt{2}}\left(T_{C}(t)+\left(C \wedge T_{C}\right)(t)\right)$

Substituting $T_{C}$ and $T_{C}$ vectors into the equations(9) and (11) we get the curve $\beta_{2}(t)$ as follow (Figure 2.):

$\beta_{2}(t)=\frac{1}{\sqrt{2}}\left(\begin{array}{l}-\cos (t) \cos (2 n t)-2 n \sin (n t) \sin (t) \cos (n t)-\frac{n}{m} \sin (t), \\ -\sin (t) \cos (2 n t)+2 n \cos (t) \cos (n t) \sin (n t)+\frac{n}{m} \cos (t), \frac{2 n}{m} \cos (n t) \sin (n t)-n\end{array}\right)$
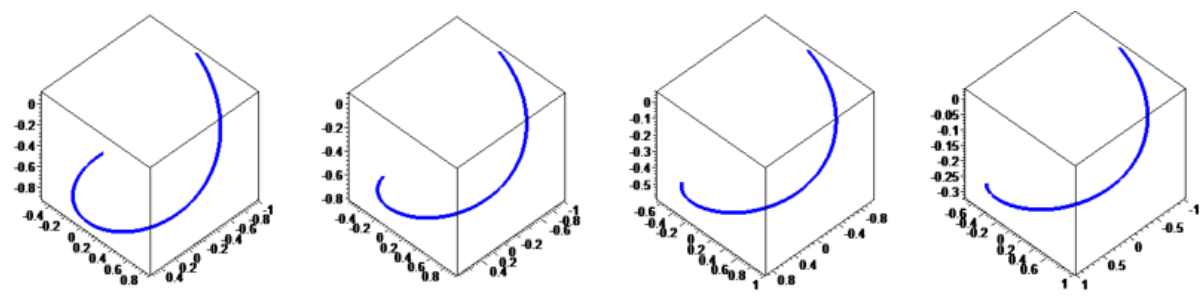

Figure 2. $\beta_{2}(t)$-Smarandache curves for $m=\frac{1}{3}, \frac{1}{5}, \frac{1}{8}, \frac{1}{16}$, respectively. 
Theorem 3.2. The geodesic curvature $K_{g}{ }^{\beta_{2}}$ according to $\beta_{2}(t)$ Smarandache curve is

$$
K_{g}^{\beta_{2}}=\frac{4 \sqrt{2}\left(\sec ^{5}(n t)+n^{2}\right)+\sqrt{2}\left(2 \sin (n t)+\cos ^{2}(n t)\right)}{\cos ^{3}(n t)\left(n^{2}+2 \sec ^{2}(n t)\right)^{\frac{5}{2}}}
$$

Proof: If we take the derivative of (22) with respect to $S$, we have

$$
\begin{aligned}
& \frac{d \beta_{2}}{d s} \frac{d s}{d t}=\frac{1}{\sqrt{2}}\left(T_{C}^{\prime}+(C \wedge T)^{\prime}\right), \\
& T_{\beta_{1}} \frac{d s}{d t}=\frac{-C-\kappa_{g} T_{C}+\kappa_{g}\left(C \wedge T_{C}\right)}{\sqrt{2}}, \\
& T_{\beta_{1}}=\frac{-C-\kappa_{g} T_{C}+\kappa_{g}\left(C \wedge T_{C}\right)}{\sqrt{1+2 \kappa_{g}^{2}}}
\end{aligned}
$$

where $\frac{d s}{d t}=\frac{\sqrt{1+2 K_{g}^{2}}}{\sqrt{2}}$. If again derivative is taken, we get

$$
\begin{aligned}
& \frac{d T_{\beta_{1}}}{d s} \frac{d s}{d t}=\frac{\left(-C-\kappa_{g} T_{C}+\kappa_{g}\left(C \wedge T_{C}\right)\right)^{\prime} \sqrt{1+2 \kappa_{g}^{2}}-\left(\sqrt{1+2 \kappa_{g}^{2}}\right)^{\prime}\left(-C-\kappa_{g} T_{C}+\kappa_{g}\left(C \wedge T_{C}\right)\right)}{1+2 \kappa_{g}^{2}} \\
& T_{\beta_{1}}^{\prime}=\frac{\sqrt{2}\left(\left(2 K_{g}{ }^{3}+K_{g}+2 K_{g} K_{g}^{\prime}\right) C-\left(2 K_{g}{ }^{4}+3 K_{g}{ }^{2}+K_{g}^{\prime}+1\right) T_{C}+\left(-2 K_{g}{ }^{4}-K_{g}{ }^{2}+K_{g}^{\prime}\right)\left(C \wedge T_{C}\right)\right)}{\left(1+2 K_{g}{ }^{2}\right)^{2}}
\end{aligned}
$$

Using the equations (6) and (25), we easily find

$$
C \wedge T_{\beta_{2}}=-\frac{K_{g}\left(T_{C}+C \wedge T\right)_{C}}{\sqrt{1+2 K_{g}^{2}}}
$$

From the equation (12), (26) and (27), the geodesic curvature of $\beta_{2}(t)$ is completed.

Definiton 3.3. Let $\alpha_{C}(t)=C(t)$ be an anti-Salkowski indicatrix curve and $\left\{C, T_{C}, C \wedge T_{C}\right\}$ be the Sabban frame of this curve. Then Smarandache curve is given by

$\beta_{3}(t)=\frac{1}{\sqrt{2}}\left(C(t)+\left(C \wedge T_{C}\right)(t)\right)$

Substituting $C$ and $C \wedge T_{C}$ vectors into the equations (7) and (11), we get the curve $\beta_{3}(t)$ as follow (Figure 3.): 
$\beta_{3}(t)=\frac{1}{\sqrt{2}}\left(\begin{array}{l}-2 \sin (n t) \cos (n t) \cos (t)+n \sin (t) \cos (2 n t)-\frac{n}{m} \sin (t), \\ -2 \sin (n t) \cos (n t) \sin (t)-n \cos (t) \cos (2 n t)+\frac{n}{m} \cos (t),-\frac{n}{m} \cos (2 n t)-n\end{array}\right)$
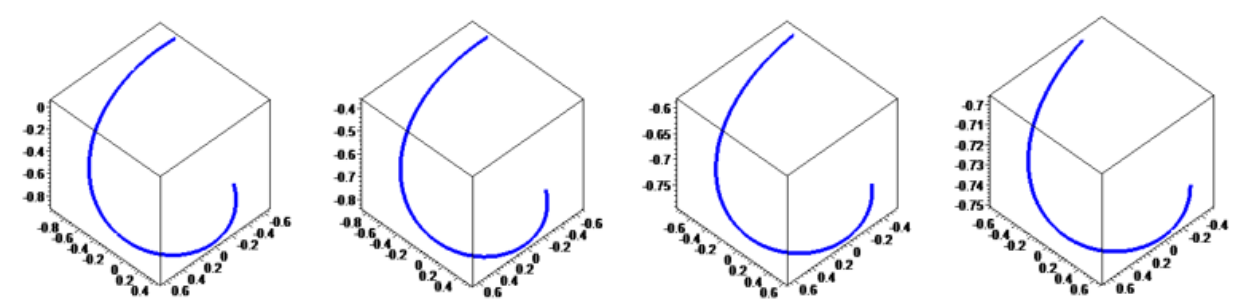

Figure 3. $\beta_{3}(t)$-Smarandache curves for $m=\frac{1}{3}, \frac{1}{5}, \frac{1}{8}, \frac{1}{16}$, respectively.

Theorem 3.3. The geodesic curvature $K_{g}{ }^{\beta_{3}}$ according to $\beta_{3}(t)$ Smarandache curve is

$$
K_{g}^{\beta_{3}}=\frac{\sqrt{2} \sec (n t)}{n-\sec (n t)}
$$

Proof: If we take the derivative of (28) with respect to $S$, we have

$$
\frac{d \beta_{3}}{d s} \frac{d s}{d t}=\frac{1}{\sqrt{2}}\left(C^{\prime}+\left(C \wedge T_{C}\right)^{\prime}\right),
$$

$T_{\beta_{3}} \frac{d s}{d t}=\frac{1-\kappa_{g}}{\sqrt{2}} T_{C}$,

$T_{\beta_{1}}=T_{C}$

where $\frac{d s}{d t}=\frac{1-K_{g}}{\sqrt{2}}$. If again derivative is taken, we get

$T_{\beta_{3}}^{\prime}=\frac{\sqrt{2}\left(-C+K_{g} C \wedge T_{C}\right)}{1-K_{g}}$.

Using the equations (6) and (32), we easily find

$$
C \wedge T_{\beta_{3}}=C \wedge T_{C}
$$

From the equation (12), (32) and (33), the geodesic curvature of $\beta_{3}(t)$ is completed.

Definiton 3.4. Let $\alpha_{C}(t)=C(t)$ be an anti-Salkowski indicatrix curve and $\left\{C, T_{C}, C \wedge T_{C}\right\}$ be the Sabban frame of this curve. Then Smarandache curve is given by

$$
\beta_{4}(t)=\frac{1}{\sqrt{3}}\left(C(t)+T_{C}(t)+\left(C \wedge T_{C}\right)(t)\right)
$$


Substituting $C, T_{C}$ and $C \wedge T_{C}$ vectors into the equations (7), (9) and (11) we get the curve $\beta_{4}(t)$ as follow (Figure 4.):

$$
\beta_{4}(t)=\frac{1}{\sqrt{3}}\left(\begin{array}{l}
\cos (2 n t)(n \sin (t)-\cos (t))-2 \sin (n t) \cos (n t)(\cos (t)+n \sin (t))-\frac{n}{m} \sin (t), \\
-\cos (2 n t)(n \cos (t)+\sin (t))+2 \sin (n t) \cos (n t)(-\sin (t)+n \cos (t))+\frac{n}{m} \cos (t), \\
\frac{n}{m}(2 \sin (n t) \cos (n t)-\cos (2 n t))-n
\end{array}\right) .
$$
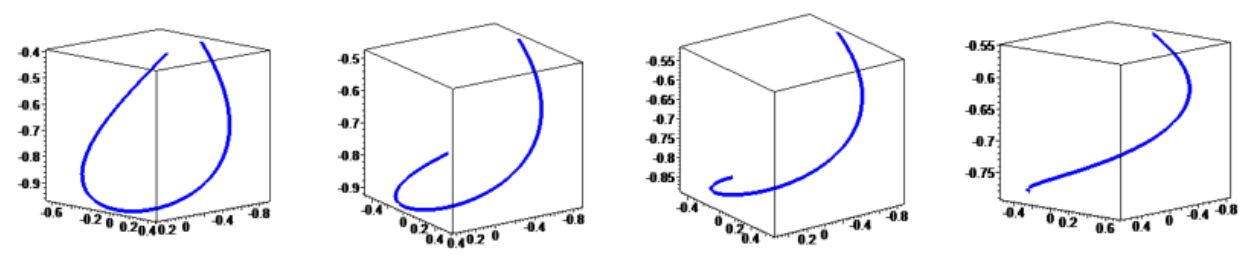

Figure 4. $\beta_{4}(t)$-Smarandache curves for $m=\frac{1}{3}, \frac{1}{5}, \frac{1}{8}, \frac{1}{16}$, respectively.

Theorem 3.4. The geodesic curvature $K_{g}^{\beta_{4}}$ according to $\beta_{4}(t)$ Smarandache curve is

$$
\begin{aligned}
K_{g}^{\beta_{4}}= & \frac{\sqrt{6}\left(2 \sec ^{5}(n t)-4 n \sec ^{4}(n t)+6 n^{2} \sec ^{3}(n t)-4 n^{3} \sec ^{2}(n t)+2 n^{4} \sec (n t)\right)}{4\left(n^{2}-n \sec (n t)+\sec ^{2}(n t)\right)^{\frac{5}{2}}} \\
& +\frac{\sqrt{6}\left(n^{5} \tan (n t)\left(\sec ^{3}(n t)-\sec ^{2}(n t)+\sec (n t)\right)\right)}{4\left(n^{2}-n \sec (n t)+\sec ^{2}(n t)\right)^{\frac{5}{2}}}
\end{aligned}
$$

Proof: If we take the derivative of (34) with respect to $S$, we have

$$
\begin{aligned}
& \frac{d \beta_{4}}{d s} \frac{d s}{d t}=\frac{1}{\sqrt{3}}\left(C^{\prime}+T_{C}^{\prime}+\left(C \wedge T_{C}\right)^{\prime}\right) \\
& T_{\beta_{4}} \frac{d s}{d t}=\frac{1}{\sqrt{3}}\left(-C+\left(1-K_{g}\right) T_{C}+K_{g}\left(C \wedge T_{C}\right)\right) \\
& T_{\beta_{4}}=\frac{1}{\sqrt{2-2 K_{g}+2 K_{g}^{2}}}\left(-C+\left(1-K_{g}\right) T_{C}(t)+K_{g}\left(C \wedge T_{C}\right)(t)\right)
\end{aligned}
$$

where $\frac{d s}{d t}=\frac{\sqrt{2-2 K_{g}+2 K_{g}^{2}}}{\sqrt{3}}$. If again derivative is taken, we get 


$$
\begin{aligned}
& \frac{d T_{\beta_{4}}}{d s} \frac{d s}{d t}=\frac{\left(-C+\left(1-K_{g}\right) T_{C}+K_{g}\left(C \wedge T_{C}\right)\right)^{\prime} \sqrt{2-2 K_{g}+2 K_{g}^{2}}}{2-2 K_{g}+2 K_{g}^{2}} \\
& -\frac{\left(\sqrt{2-2 K_{g}+2 K_{g}^{2}}\right)^{\prime}\left(-C+\left(1-K_{g}\right) T_{C}+K_{g}\left(C \wedge T_{C}\right)\right)}{2-2 K_{g}+2 K_{g}{ }^{2}} \\
& T_{\beta_{4}}^{\prime} \frac{d s}{d t}=\frac{2 K_{g}^{3}-4 K_{g}^{2}+2 K_{g} K_{g}^{\prime}-K_{g}^{\prime}+4 K_{g}-2}{2 \sqrt{2}\left(1-K_{g}+K_{g}^{2}\right)^{\frac{3}{2}}} C \\
& +\frac{-2 K_{g}^{4}+2 K_{g}^{3}-4 K_{g}^{2}-K_{g} K_{g}^{\prime}-K_{g}^{\prime}+2 K_{g}-2}{2 \sqrt{2}\left(1-K_{g}+K_{g}^{2}\right)^{\frac{3}{2}}} T_{C} \\
& +\frac{-2 K_{g}^{4}+4 K_{g}^{3}-4 K_{g}^{2}-K_{g} K_{g}^{\prime}+2 K_{g}^{\prime}+2 K_{g}}{2 \sqrt{2}\left(1-K_{g}+K_{g}^{2}\right)^{\frac{3}{2}}} C \wedge T_{C} \\
& T_{\beta_{4}}^{\prime}=\frac{\sqrt{3}\left(2 K_{g}^{3}-4 K_{g}^{2}+2 K_{g} K_{g}^{\prime}-K_{g}^{\prime}+4 K_{g}-2\right)}{4\left(1-K_{g}+K_{g}{ }^{2}\right)^{2}} C \\
& +\frac{\sqrt{3}\left(-2 K_{g}^{4}+2 K_{g}^{3}-4 K_{g}^{2}-K_{g} K_{g}^{\prime}-K_{g}^{\prime}+2 K_{g}-2\right)}{4\left(1-K_{g}+K_{g}{ }^{2}\right)^{2}} T_{C} \\
& +\frac{\sqrt{3}\left(-2 K_{g}^{4}+4 K_{g}^{3}-4 K_{g}^{2}-K_{g} K_{g}^{\prime}+2 K_{g}^{\prime}+2 K_{g}\right)}{4\left(1-K_{g}+K_{g}^{2}\right)^{2}} C \wedge T_{C}
\end{aligned}
$$

Using the equations (6) and (36), we easily find

$$
C \wedge T_{\beta_{4}}=\frac{-K_{g} T_{C}+\left(1-K_{g}\right)\left(C \wedge T_{C}\right)}{\sqrt{2} \sqrt{1-K_{g}+K_{g}{ }^{2}}}
$$

From the equation (12), (37) and (38), the geodesic curvature of $\beta_{4}(t)$ is completed.

\section{References}

Fenchel, W. (1951). On the differential geometry of closed space curves. Bulletin of the American Mathematical Society, 57, 44-54.

Gür, S. and Şenyurt, S. (2010). Frenet vectors and geodesic curvatures of spherical indicators of Salkowski curve in $\mathrm{E}^{\wedge} 3$. Hadronic Journal, 33(5), 485.
Koenderink, J. (1990). Solid shape. MIT Press, ISBN 978-0-262-11139-3, $715 \mathrm{p}$.

Monterde, J. (2009). Salkowski curves revisited: a family of curves with constant curvature and non-constant torsion Computer Aided Geometric Design, 26, 271-278. https://doi.org/10.1016/j.cagd.2008.10.002

Sabuncuoğlu, A. (2006). Diferensiyel geometri. Nobel yayınları 258, ISBN 975-591-237- 1, Ankara Türkiye, 440s. 
Salkowski, E.L. (1909). Zur transformation von raumkurven. Mathematisch Annalen, 4(66), 517557.

Şenyurt, S. and Öztürk, B. (2018). Smarandache curves of Salkowski curve according to Frenet frame. Turkish Journal of Mathematics and Computer Science, 10, 190-201.

Şenyurt, S. and Öztürk, B. (2018). Smarandache curves of anti-Salkowski curve according to Frenet frame. Proceedings of the International Conference on Mathematical Studies and Applications (ICMSA), October 2018, Karaman, Turkey, pp.132-143.

Uzun, M. and Şenyurt, S. (2020). Smarandache curves according to Sabban frame generated by the spherical indicatrix curves of the unit darboux vector of Salkowski curve. Journal of the Institute of Science and Technology, 10(3), 19661974. https://doi.org/10.21597/jist.703495 